\title{
Simulation of sand particle transport by coupled CFD-DEM: First investigations
}

\author{
Désirée Plenker \\ Institute of Geotechnical Engineering and Construction Management \\ Hamburg University of Technology, Hamburg, Germany \\ Jürgen Grabe \\ Institute of Geotechnical Engineering and Construction Management \\ Hamburg University of Technology, Hamburg, Germany
}

\begin{abstract}
The continuum based Euler-Euler approach represents the main field of application for the simulation of sediment transport processes. Herein, the decisive phases of free water and soil are modelled by interpenetrating continua. Although mixing of the phases is possible, the multi-component character of the soil phase, as a mixture of solid grains and pore water, is neglected. Hence, a coupling between the free water and the pore water remains unnoticed as well. However, this coupling represents an important factor for determining the current state of the soil boundary near the transition zone. Due to water level changes caused by ship induced bow and stern waves, excess pore water pressure can occur in the upper soil layers. As a result, fluidisation effects can be initiated, which reduce the erosion resistance. To consider these fluidisation effects, the soil has to be treated as a mixture of dispersed grain particles and pore water by a Lagrange-Euler approach. The coupling of the Discrete Element Method (DEM) and the Computational Fluid Dynamics (CFD) approach offers this possibility. Thereby, the DEM is used for modelling the dispersed particles of the Lagrangian regime, while the CFD method models the continuum Euler-phase of the water. This paper introduces the coupled CFD-DEM method for simulating sand particle transport at the boundary layer transition zone. The model investigations and first results of simulations regarding the initiation of motion are presented.
\end{abstract}

\section{INTRODUCTION}

Sediment transport processes are characterized by a high number of influencing parameters including even highly dynamic processes. Due to this, a characterisation of the transport phenomena through exact analytical solutions remains unformed. However, several empirical models, which are based on the knowledge of numerous physical model as well as field tests, allow an appropriate solution for most practical applications. These models are used to estimate of transport rates as well as for numerical simulations of sediment transport on diverse spatial and temporal scales. In numerical simulations the involved phases, in particular water and bed material, are approximated by continua as an Computational Fluid Dynamics (CFD) Euler-Euler approach. Thereby, the multicomponent character of the bed material is neglected. As a consequence, several effects can not be captured. One of these effects is fluidisation due to excess pore water pressure caused by surge waves due to, for instance, ship passages. To consider these flu- idisation effects, a interaction between pore and free water has to be enabled. Furthermore, the grain skeleton must be able to disperse under the influence of excess pore water pressures. The Discrete Element Method (DEM) offers the possibility, to model grains as dispersed spherical particles. The particle transport and interaction is considered by contact models and force-displacement laws. Beside the interaction among each other, the particles at the transition zone are affected by the interaction with the surrounding water. The coupled CFD-DEM method allows the possibility to take the interaction effects into account. While the DEM is used for the simulation of the particles, the CFD represents the fluid phase. Due to a coupling of both methods, phase specific informations can be exchanged and thus, be used for the calculation of interaction forces. Furthermore, the coupled CFD-DEM method is able to simulate transport processes in a more detailed way than in common CFD approaches. Since the CFD-DEM is adopted on a microscopic scale, even the detaching of individual grains and proximate transportation as bed load 
or suspended load can be modelled. Due to this, the CFD-DEM could be applied to gather more detailed informations to specific flow processes. Hereby, field measurements or physical model test can be supplemented. In this paper the coupled CFD-DEM approach will be introduced in sediment transport applications. Thereby, the concerning methods and first model investigations will be presented. Furthermore, results of first simulations for the initiation of sediment transport are described and discussed.

The applied software for the applied simulations is the open source software CFDEMcoupling. This software offers the coupling of the DEM code LIGGGHTS and the CFD software library OpenFOAM. LIGGGHTS is an advanced code of the molecular dynamics code LAMMPS, which has been improved for granular assemblies (Kloss and Goniva 2011).

\section{CFD-DEM METHOD}

The CFD-DEM method represents the combination of two different numerical approaches: On the one hand the CFD method with an Eulerian continuum approach and on the other hand the DEM method with a Lagrangian discrete approach. Thereby, each method solves the governing equations for the appropriate phase in the simulation domain independently. By coupling the two methods, a interaction of both phases in the simulation domain is enabled and informations of one phase are transferred to the other phase. In doing so, the phases are able to interact. In the following sections the governing equations of the particular methods as well as the coupling procedure are introduced.

\subsection{CFD - Governing Equations}

The CFD method is a common tool for simulating hydrodynamic processes. Thereby, CFD does not represent a specific method rather than a collection of different numerical approaches for the simulation of fluid flows. The fundamental basis of all these methods are Navier-Stokes equations. The Navier-Stokes equations describe the motion of a viscous fluid by conservation equations of mass and momentum. Since these equations consist of a system of partial differential equations, which cannot be solved analytically, numerical discretisation schemes are needed. A common tool in CFD for spatial discretisation is the Finite Volume Method (FVM), which is used in the conducted simulations. The FVM approximates the modelled area by three-dimensional discrete control volume elements, with a integration point in the elements cell center. Furthermore, the fluxes over the element edges are summed up, to guarantee a consistent solution.

The CFD in OpenFOAM gives different solutions for the simulation of multiphase flows. Most methods are applicable for the simulation of continua as fluids or gases but are not suitable for the simulation of solid mixtures like particle flow or soil, consisting of dispersed particles and pore fluid. These mixtures can be approximated as a continuum by constitutive equations, describing the behaviour of the solid. Thereby, the dispersed character of the mixture as well as the fact that the different phases are able to interpenetrate are neglected. The Two Fluid Modelling (TFM) method is a CFD method which is able to simulate a rudimental dispersed behaviour. The TFM provides two interpenetrating continua, with one of them consisting of discrete particles. By expansion of the TFM with the Kinetic Theory of Granular Flow (KTGF) the particle properties as well as their interaction is considered ( $\mathrm{Lu}$ et al. 2015). Furthermore, the conservative equations for mass and momentum are extended for the multiphase and interpenetrational application of the TFM.

This approach is transferred to conservative equations of the fluid phase as part of the CFD-DEM method. Equation 1 describes the conservative equation for mass, while equation 2 represents the equation system for the conservation of momentum.

$$
\begin{aligned}
& \frac{\partial}{\partial t}\left(\alpha_{\mathrm{f}} \rho\right)+\nabla \cdot\left(\alpha_{\mathrm{f}} \rho \mathbf{v}\right)=0 \\
& \frac{\partial}{\partial t}\left(\alpha_{\mathrm{f}} \rho \mathbf{v}\right)+\nabla \cdot\left(\alpha_{\mathrm{f}} \rho \mathbf{v} \mathbf{v}\right)= \\
& \\
&-\alpha_{\mathrm{f}} \nabla p+\nabla \cdot\left(\alpha_{\mathrm{f}} \boldsymbol{\tau}_{\mathrm{f}}\right)+\alpha_{\mathrm{f}} \rho \mathbf{g}+R_{\mathrm{pf}}
\end{aligned}
$$

where $\rho$ is the fluid mixture density, $\mathbf{v}$ is the fluid mixture velocity, $p$ is the pressure and $\tau_{f}$ is the shear tensor of the fluid phase. This version looks quite similar compared to the original Navier-Stokes equations. The only difference is the factor $\alpha_{\mathrm{f}}$ and the summand $R_{\mathrm{pf}}$ in the transport equations. The factor $\alpha_{\mathrm{f}}$ describes the volume fraction of fluid in a cell. $R_{\mathrm{pf}}$ represents a force exchange term between the fluid and the particle phase. This term governs the coupling of the two phases.

\subsection{DEM - Governing Equations}

The DEM was developed by Cundall in 1971 for applications in rock mechanics for the simulation of three-dimensional body collisions. This approach allows the simulation of a large number of dispersed particle bodies in a Lagrangian formulation. Thus, the simulation of highly dynamic particle flows with large displacements is possible.

In DEM the trajectories of each particle are calculated by consideration of external forces. Therefore, the current forces acting on a single particle are summed up. Through the application of Newton's second law the resulting translational (see Equ. 3) and rotational (see Equ. 4) velocities for the next time step 
are calculated (Cundall and Strack 1979).

$$
\begin{aligned}
m_{\mathrm{p}} \frac{d}{d t} \mathbf{v}_{\mathrm{p}} & =\sum \mathbf{F}_{i} \\
I_{\mathrm{p}} \frac{d}{d t} \boldsymbol{\omega}_{\mathbf{p}} & =\sum \mathbf{T}_{i}
\end{aligned}
$$

where $m_{\mathrm{p}}$ represents the particle mass, $\mathbf{v}_{\mathrm{p}}$ the particle phase velocity, $\mathbf{F}_{i}$ the sum of forces (contact forces and body forces), $I_{\mathrm{p}}$ the moment of inertia, $\omega_{\mathbf{p}}$ the particle angular velocity and $\mathbf{T}_{i}$ the sum of torques.

In addition to the calculation of particle trajectories, the DEM contains a solution for particle contact force detection. The first contact formulation of Cundall was based on the velocity detection of single rigid particle collisions (Stein et al. 2004). Since this formulation was limited to single collisions and hence was appropriate only for very dilute particle flows, this initial formulation was later extended by Cundall and Strack (1979). This new formulation improves the simulation of particle collisions by assuming the particles as deformable bodies. In contrast to an expensive simulation of the particle deformation due to an impact event, the so called soft sphere model approximates the deformation by an overlap of the interacting particles (Cundall and Hart 1992). As a result, the handling of multi sphere contacts and treatment of dense packings is possible ( $\mathrm{Lu}$ et al. 2015). This modern approach is used in the most common DEM codes.

In the soft sphere model the particle overlap is denoted as the particle displacement $\delta_{i}$. For the calculation of contact forces, a force-displacement law or contact law is applied. This contact law consists of several interconnected rheological elements, each representing a different material behaviour like idealelasticity (spring), ideal-viscosity (dashpot) or idealplasticity (friction-slider). On the basis of these models the contact forces are calculated. In the conducted simulations the particle contact forces are differentiated into a normal $\mathbf{F}_{p, n}$ and a tangential $\mathbf{F}_{p, t}$ contact force component. Thereby, each component consists of a spring-dashpot combination, which represents viscoelastic behaviour (see figure 1). The normal and tangential components of the contact forces are determined by

$$
\begin{aligned}
& \mathbf{F}_{\mathrm{p}, n}=k_{n} \boldsymbol{\delta}_{n}-c_{n} \Delta \mathbf{v}_{\mathrm{p}, n} \\
& \mathbf{F}_{\mathrm{p}, t}=k_{t} \boldsymbol{\delta}_{t}-c_{t} \Delta \mathbf{v}_{\mathrm{p}, t}
\end{aligned}
$$

with $k_{n}$ and $k_{t}$ representing the spring stiffness, $c_{n}$ and $c_{t}$ representing the damping coefficients and $\Delta \mathbf{v}_{\mathrm{p}, n}$ and $\Delta \mathbf{v}_{\mathrm{p}, t}$ representing the relative particle velocities in normal and tangential direction respectively.

For the determination of the contact coefficients the Hertz-Mindlin and Deresiewicz model is applied in the conducted simulations. Thereby, the normal spring stiffness $k_{n}$ is determined by Hertz's contact
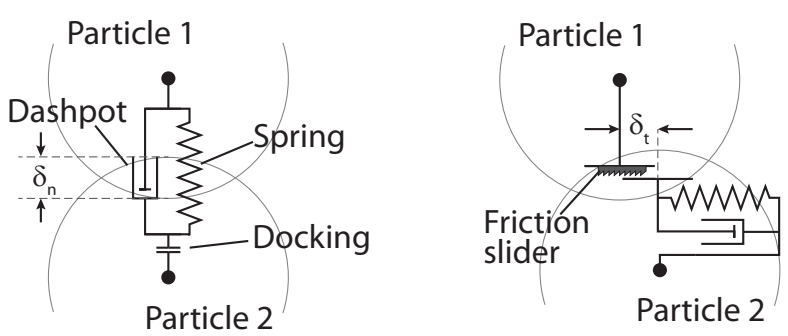

Figure 1: Rheological scheme of normal contact (left) and tangential contact (right) of two colliding particles (from Plenker (2015))

theory (Hertz 1881), while the spring stiffness $k_{t}$ is calculated on basis of Mindlin and Deresiewicz (1953). For the calculation of the damping coefficients $c_{n}$ and $c_{t}$ a non-linear damping term of Tsuji et al. (1992) is applied (Hu et al. 2011).

\subsection{Coupled CFD-DEM}

The coupled CFD-DEM method is an established method in process engineering. The simulation of flows and fluidisation processes of granular particles in a gas or fluid phase is a common implementation (Crowe et al. 2012). The CFD-DEM method is based on the coupling of two self-sufficient methods of CFD and DEM approach, already discussed above. Each method calculates the governing equations for the representing phase independently. Though, the coupling allows a communication between the two programs and an exchange of relevant data for the calculation of the interaction, like particle sizes, positions, velocities and resulting interaction forces.

The calculation of interaction is mainly affected by the ratio between the size of the CFD cells and the size of the DEM particles. Mainly, two concepts are available. On one hand, the resolved method, where the particles are significantly larger than the CFD cells (see figure 2 a). This leads to a very high resolution of the simulation domain and very accurate calculation of the interaction between an individual particle and the surrounding fluid. On the other hand, the unresolved method, where the CFD cells are equal or larger than the DEM particles (see Fig. 2 b). Thereby, the values of the individual particles located in a CFD cell are calculated and then averaged before the data is exchanged to CFD. This leads to less a precise solution than in the resolved method.

However, the CFD-DEM is strongly limited to small model scales regarding granular phases with a small particle diameter. Even for simulation domains of a few centimetres, millions of particles are necessary. These dimensions can not be examined by the resolved method, since the computational costs would be enormous. The unresolved method is a reasonable compromise for the simulation of dispersed granular material. 


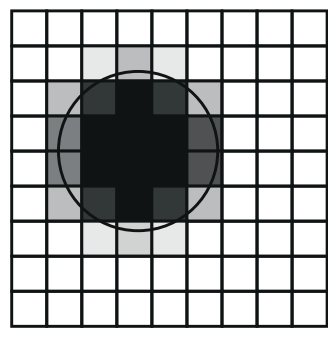

a) resolved

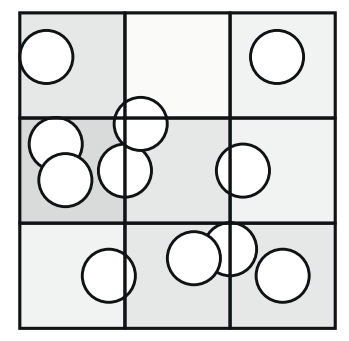

b) unresolved
Figure 2: Concept of resolved (left) and unresolved (right) CFDDEM application. The cell colouring corresponds with the volume fraction of containing particles (from (Plenker 2015))

\subsubsection{Fluid-particle interaction forces}

In CFD-DEM the phase interaction is modelled by the approach of interaction forces. The main interaction forces between fluid and particles are the drag force $F_{p, D}$, the pressure gradient force $F_{p, p}$, the virtual mass force $\mathbf{F}_{\mathrm{p}, \mathrm{VM}}$, the Basset force $\mathbf{F}_{\mathrm{p} \text {,hist }}$, which is also known as the history force, and the dynamic lift forces such as the Saffmann $\mathbf{F}_{\mathrm{p}, \mathrm{L}, \mathrm{Saff}}$ and the Magnus force $\mathbf{F}_{\mathrm{p}, \mathrm{L}, \mathrm{Magn}}$. Further information to the specific forces can be found in (Zhou et al. 2010), (Zhu et al. 2007) and (Crowe et al. 2012). Table 1 lists the applied force models of the conducted simulations. The drag force $\mathbf{F}_{\mathrm{p}, \mathrm{D}}$ is the most significant force regarding particle flow in a viscous fluid. Accordlingly, a large number of force models exist in literature. For the following simulations the drag model of Koch and Hill (2001) is applied. According to Bokkers et al. (2004), this model lead to better results for intermediate volume fractions than the often used Gidaspow (1994) approach. A lift force $\mathbf{F}_{\mathrm{p}, \mathrm{L}}$ is considered by the extended Saffman analysis of Mei (1992). Additionally, the model formulation of McLaughlin (1991) is added with a correction factor for the lift coefficient $C_{\mathrm{L}}$. The Magnus component of the lift forces is neglected. The virtual mass force $\mathbf{F}_{\mathrm{p}, \mathrm{VM}}$ is applied on the basis of Odar and Hamilton (1964).

In contrast to the examined forces, the consideration of the pressure gradient force $\mathbf{F}_{\mathrm{p}, \mathrm{p}}$ depends on the chosen governing equations in the continuum CFD method. Mainly two different formulations of the conservative equation of momentum exists. In the first formulation, the so called model $\mathrm{A}$, the pressure gradient term is shared between the particle and the fluid phase. The second formulation, model B, the pressure term is recognized just on the fluid side (Gidaspow 1994). Another difference between the formulations is the consideration of viscous particle-fluid interaction forces. While model B contains viscous forces, model A is only applicable on the absence of viscous stresses (Zhou, Kuang, Chu, \& Yu 2010). Since model $\mathrm{A}$ is applied here, the interaction forces due to pressure gradients $\mathbf{F}_{\mathrm{p}, \mathrm{p}}$ and viscous forces $\mathbf{F}_{\mathrm{p}, \nu}$ has to be added additionally to the incorporated forces.

\subsubsection{Exchange of interaction forces}

The interaction forces between fluid and particle are recognized on the CFD as well on the DEM side. On the CFD side the interactions forces are added by a momentum exchange (Goniva et al. 2012). As described in section 2.1, the conservation equation of momentum contains an additional force exchange term $R_{\mathrm{pf}} \cdot R_{\mathrm{pf}}$ is given as

$$
R_{\mathrm{pf}}=K_{\mathrm{pf}}\left(\mathbf{v}-\mathbf{v}_{\mathrm{p}}\right)
$$

with the interaction force coefficient $K_{\mathrm{pf}} . K_{\mathrm{pf}}$ is the superposition of the interaction forces from drag, pressure gradient, viscosity, lift as well as virtual mass. Thereby, the implemented and in $K_{\mathrm{pf}}$ included interaction forces must harmonize, with the applied formulation model for the conservation equation of momentum in CFD.

On DEM side the interaction forces are added to the sum of forces and torques in Newton's second law. Hence, $\sum \mathbf{F}_{i}$ consists of the normal and tangential contact forces $\mathbf{F}_{\mathrm{p}, n}$ and $\mathbf{F}_{\mathrm{p}, t}$, the particle weight $\mathbf{F}_{\mathrm{p}, g}$ and the fluid-particle interaction forces (see Equ. 8).

$$
\begin{aligned}
\sum \mathbf{F}_{i}= & \mathbf{F}_{\mathrm{p}, n}+\mathbf{F}_{\mathrm{p}, t}+\mathbf{F}_{\mathrm{p}, g}+\mathbf{F}_{\mathrm{p}, \mathrm{D}} \\
& +\mathbf{F}_{\mathrm{p}, \mathrm{L}}+\mathbf{F}_{\mathrm{p}, \mathrm{p}}+\mathbf{F}_{\mathrm{p}, \nu}+\mathbf{F}_{\mathrm{p}, \mathrm{VM}}
\end{aligned}
$$

\section{INITIATION OF SEDIMENT TRANSPORT}

Sediment transport is subject to numerous and transient influences, highly dynamic flow as well as local turbulences at the boundary layer on micro scale. Due to this complexity, a detailed examination and analytical description of the processes at the transition zone is nearly impossible. Nevertheless, empirical models exist which allow a prediction of transport amounts and the estimation of erosion or sedimentation phenomena. These empirical models are developed on the basis of monitoring in field or physical model tests. The basic principle behind the models is the identification of motion at the river bed. The initiation of motion is characterized by an ultimate limit state or critical state, where the hydrodynamic load equals the resistance of the granular structure of the bed. If the hydrodynamic load exceeds this critical state, sediment transport occurs.

A great number of sediment transport models exist. Besides the different characterisations of the hydrodynamic regime or the river bed, the models can be classified into two main groups by the definition of the critical state.

The first group uses velocities, as parameters for the identification of the critical state. The most famous representation of this approach is the theory of Hjulström. Hjulström (1935) developed the well known diagram, which was later extended by i.a. Åke Sundborg (see Figure 3). In this diagram a medium 


\begin{tabular}{|c|c|c|}
\hline Drag force & $\begin{array}{l}\mathbf{F}_{\mathrm{p}, \mathrm{D}}=\frac{V_{\mathrm{p}} \beta}{\alpha_{\mathrm{p}}}\left(\mathbf{v}-\mathbf{v}_{\mathrm{p}}\right) \\
\beta=\frac{18 \nu_{\mathrm{f}} \alpha_{\mathrm{f}} \alpha_{\mathrm{p}}}{d_{\mathrm{p}}^{2}} \mathbf{F}_{\beta} \\
\mathbf{F}_{\beta}\left(\alpha_{\mathrm{p}}\right)=\alpha_{\mathrm{f}}\left(\mathbf{F}_{0}\left(\alpha_{\mathrm{p}}\right)+\frac{1}{2} \mathbf{F}_{3}\left(\alpha_{\mathrm{p}}\right) \operatorname{Re}_{\mathrm{p}}\right) \\
\quad\left(\alpha_{\mathrm{p}}<0,4\right) \rightarrow \mathbf{F}_{0}\left(\alpha_{\mathrm{p}}\right)=\frac{1+3\left(\alpha_{\mathrm{p}} / 2\right)^{1 / 2}+(135 / 64) \alpha_{\mathrm{p}} \ln \left(\alpha_{\mathrm{p}}\right)+16.14 \alpha_{\mathrm{p}}}{1+0.681 \alpha_{\mathrm{p}}-8.48 \alpha_{\mathrm{p}}^{2}+8.16 \alpha_{\mathrm{p}}^{3}} \\
\quad\left(\alpha_{\mathrm{p}}>0,4\right) \rightarrow \mathbf{F}_{0}\left(\alpha_{\mathrm{p}}\right)=\frac{10 \alpha_{\mathrm{p}}}{\left(1-\alpha_{\mathrm{p}}\right)^{3}} \\
\mathbf{F}_{3}\left(\alpha_{\mathrm{p}}\right)=0.0673+0,212 \alpha_{\mathrm{p}}+0.0232\left(1-\alpha_{\mathrm{p}}\right)^{5}\end{array}$ & (Koch and Hill 2001) \\
\hline Pressure force & $\mathbf{F}_{\mathrm{p}, \mathrm{p}}=-V_{\mathrm{p}}(\nabla p)$ & \\
\hline Viscous force & $\mathbf{F}_{\mathrm{p}, \nu}=-V_{\mathrm{p}}(\nabla \cdot \boldsymbol{\tau})$ & \\
\hline Virtual mass force & $\mathbf{F}_{\mathrm{p}, \nu}=\frac{1}{2} \rho V_{\mathrm{p}} \frac{\partial\left(\mathbf{v}-\mathbf{v}_{\mathrm{p}}\right)}{\partial t}$ & (Odar and Hamilton 1964) \\
\hline Lift force & $\begin{array}{l}\mathbf{F}_{\mathrm{p}, \mathrm{L}}=\frac{1}{8} C_{\mathrm{L}} \rho \mathbf{v}_{\mathrm{p}}^{2} \pi d_{p}^{2} \\
C_{\mathrm{L}}=J(\epsilon) \cdot 4.11 \cdot \epsilon \\
\epsilon=\frac{\sqrt{R e_{G}}}{R e_{p}} ; R e_{p}=\frac{\left(\mathbf{v}-\mathbf{v}_{\mathrm{p}}\right) \cdot d_{p}}{\nu} ; R e_{G}=\frac{d \mathbf{v}}{d t} \frac{d_{p}^{2}}{\nu} \\
\quad(\epsilon<1) \rightarrow J(\epsilon)=-140 \epsilon^{5} \lg \left(\epsilon^{-2}\right) \\
\quad(1 \leq \epsilon \leq 20) \rightarrow J(\epsilon)=0.3(1+\tanh (2.5 \cdot \lg (\epsilon+0.191))) \\
\quad(0.667+\tan (6(\epsilon-0.32))) \\
\quad(\epsilon>20) \rightarrow J(\epsilon)=1-0.287 \epsilon^{-2}\end{array}$ & $\begin{array}{l}\text { (Mei 1992) } \\
\text { (McLaughlin 1991) }\end{array}$ \\
\hline
\end{tabular}

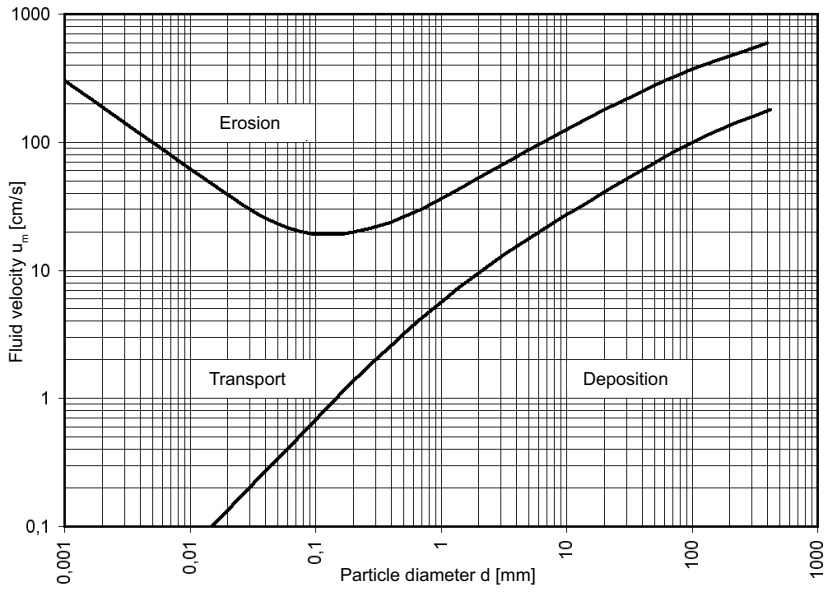

Figure 3: Hjulström diagram (Source: geocaching.com)

flow velocity $u_{m}$ is plotted against the particle diameter $d$. The diagram area is divided into three zones, representing the condition states of erosion, transport and deposition. With $d$ as input parameter, the critical velocity for the initiation of motion or deposition can be determined.

The simplicity of this is approach is very attractive, since the informations can be extracted easily. However, according to Hjulström (1935) this approach is only valid for loosely deposited and homogeneous bed material. Furthermore, the diagram is only appropriate for an approximate estimation of transport, since many influencing parameters, describing soil resistance or flow turbulences, are neglected (Dittrich 1998).

The second group of sediment transport models represents the approach of critical shear stresses. This approach was first developed by Shields (1936). Shields considerations based on the balance of forces on a single grain at the bed layer. As a result he expressed the initiation of motion as a dimensionless ratio of the critical bed shear stress $\tau_{c r}$, mostly interpreted as the bed shear stress $\tau_{0}$, and the submerged weight of a grain (Buffington 1999). This dimensionless number was later declared as the Shield's parameter $\Theta$ or $F r^{*}$.

$$
F r^{*}=\Theta=\frac{\tau_{0}}{\left(\rho_{p}-\rho\right) \cdot g \cdot D}
$$

where $D$ represents the characteristic grain diameter. Due to several model tests Shields generated his famous diagram on the basis of $\mathrm{Fr}^{*}$ as a function of the dimensionless particle Reynolds number $R e_{*}$.

$$
R e_{*}=\frac{v_{c r} D}{\nu}
$$

with the characteristic velocity $v_{c r}$. Shields recommends the criteria of Kramer (1935) for the visual observation of incipient motion. Kramer (1935) differentiated four levels of bed movement: No movement, weak movement, medium movement and general movement. The level of weak movement represents motion of only small particles at isolated spots. The medium movement level denote the transport of particle with a mean diameter without transportation of significant material quantity. The last level represents the condition where even the largest particles are in motion. The bed configuration is significantly affected at this stage of motion (Buffington 1999). 


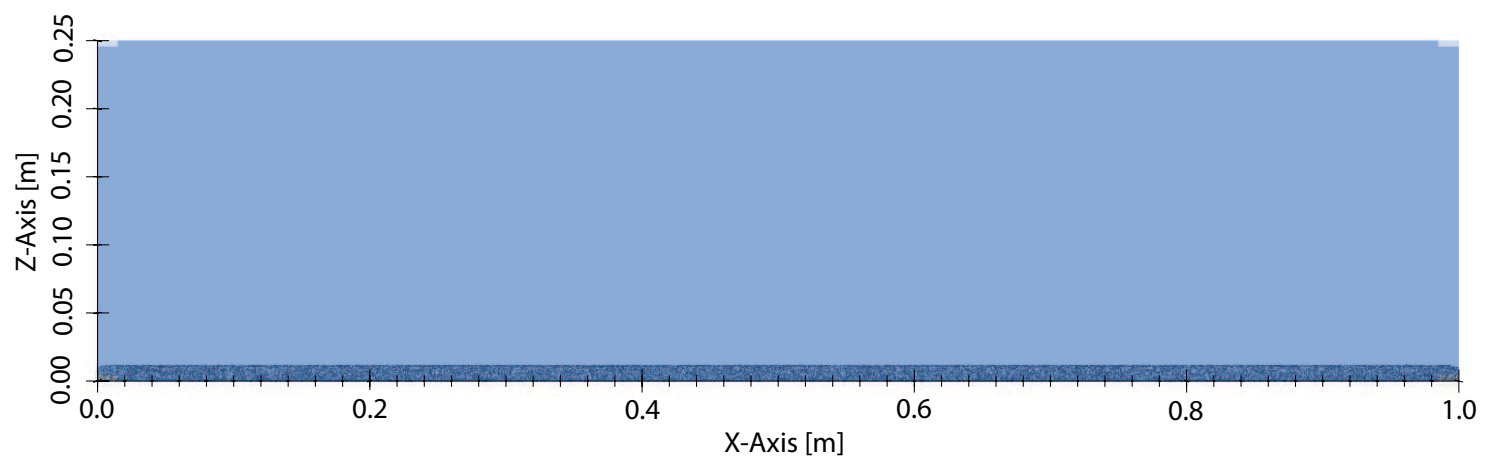

Figure 4: Profile of the simulation domains

The approach of critical shear stresses based on Shields and several supplemental research, represents the most applied basic principle for sediment transport investigations (Dittrich 1998). Since this approach derives from semi empiric considerations, it is an improvement to the method of Hjulström. However, even the approach of Shields shows weaknesses in specific applications. According to Zanke (2001), the critical shear stresses are overestimated for small particle Reynolds numbers.

In current research the influence of turbulence, serves as a central point for the initiation of particle movement. Thereby, the initiation of motion is no longer expressed by the mean shear stress $\bar{\tau}$, but by the sum of $\bar{\tau}$ and an additional shear stress fluctuation $\tau^{\prime}$ (Zanke 2003). Additionally, a turbulence induced lift force due to turbulent pressure fluctuations is considered (Zanke 2001). The full model approach as well as a transmission to the Shields diagram is included in Zanke (2003).

\section{MODEL INVESTIGATION}

The initiation of motion serves as an issue for the first model applications. The initial setup consists of a narrow, water filled channel with a small particle layer of $1 \mathrm{~cm}$ height. In the simulations, an overflow over the particle layer in positive $\mathrm{x}$-direction is induced. By a slow stepwise increase of the flow velocity, the critical point of particle transport will be determined.

As mentioned in section 2, the fluid and the particle phase are treated by independent methods and codes. As a result, an individual simulation domain for each phase has to be employed. Even though, the domain dimensions shall correlate, fundamental phase boundary conditions, like the definition of walls, can deviate. The applied boundary conditions and properties of each phase are introduced separately in the following passages.

\subsection{CFD - Fluid phase}

The simulation domain and its dimensions is shown in figure 4. The channel has a length (x-axis) of $1 \mathrm{~m}$, a height (z-axis) of $0.25 \mathrm{~m}$ and a width (y-axis) of
$0.02 \mathrm{~m}$. The length of the simulation domain is substantiated by the initiation of a realistic boundary layer velocity distribution for the particle transport analysis. According to figure 5, the inlet condition is assumed as an uniform velocity distribution with $v_{\text {inlet }}$, to permit a stepwise increase of the flow velocity in a simple manner. To develop the boundary layer velocity ditribution, the upstream area of $0.9 \mathrm{~m}$ is applied as a initiation region. The inlet condition is devided into two parts, whereby the upper part represents the mentioned uniform velocity distribution (see figure 5). The lower part, according to the later height of the particle layer, is adjusted to align freely while preventing backflow. This condition is also adopted for the outflow boundary surface.

The y- and z-normal surfaces are assumed to be rigid walls, which are impermeable. Thereby, the ynormal and the upper z-normal surface sustain slipconditions to avoid a interference on the velocity distribution. The lower z-normal surface is assumed as a rough wall with a roughness, according to the sand roughness of Nikuradse, appropriate to the applied DEM particle diameter.

The fluid properties are presumed for water at temperature of $20^{\circ} \mathrm{C}$ (see table 2 ).
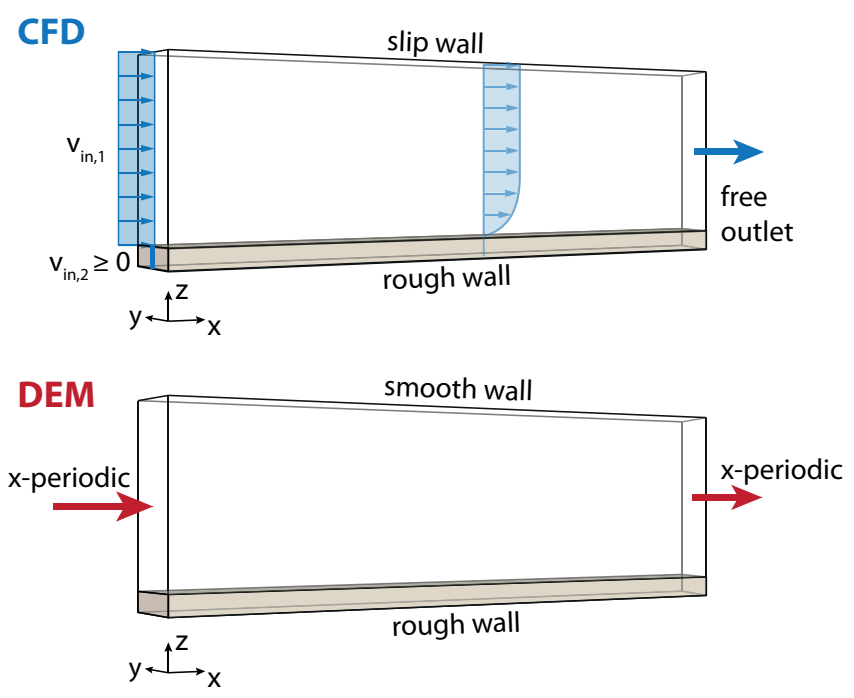

Figure 5: Sketch of the CFD and DEM simulation domains with representative boundaries 
Table 2: Fluid properties of the CFD simulation

\begin{tabular}{lrl}
\hline Parameters & & \\
\hline Density $\rho_{f}$ & 1000 & $\mathrm{~kg} / \mathrm{m}^{3}$ \\
kinematic viscosity $\nu_{\mathrm{f}}$ & $1 \cdot 10^{-6}$ & $\mathrm{~m}^{2} \mathrm{~s}^{-1}$ \\
\hline
\end{tabular}

\subsection{DEM - Particle phase}

The particle layer is established in an initial simulation as uniform particles of a specified diameter, generated over the lower half volume of the simulation area. The particles are made to deposit due to gravity. After accomplishing a steady state, particles above a height of $1 \mathrm{~cm}$ are eliminated. Thus, a very smooth particle layer surface is attained. A detailed view of the particle layer at the simulation domain inlet area is given in figure 6 . It shows a mono-dispersed packing with a particle diameter of $0.8 \mathrm{~mm}$. Further, simulations with a particle diameter of $0.6 \mathrm{~mm}$ and $0.9 \mathrm{~mm}$ were conducted.

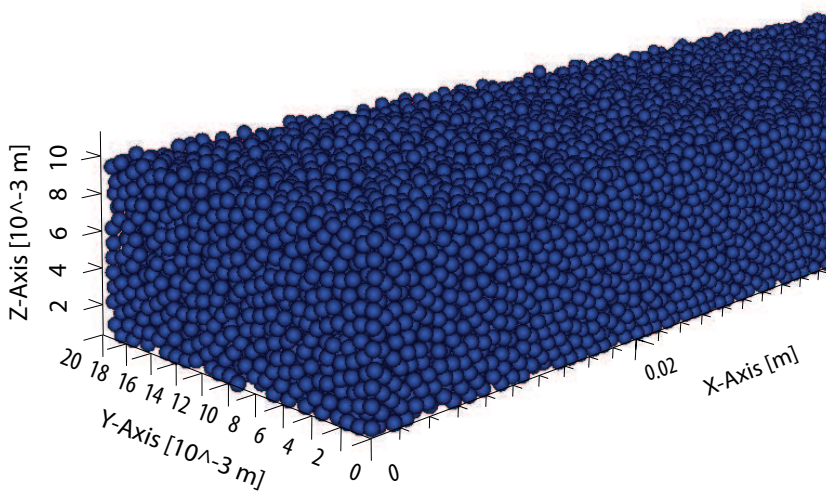

Figure 6: Detail of the particle layer at the simulation domain inlet $\left(d_{p}=8 \mathrm{~mm}\right)$

According to figure 5, the upper and lower $\mathrm{z}$ normal surfaces of the DEM domain are assumed as walls. Thereby, the upper wall receives parameters for a smooth surface, while the lower wall receives properties according to the particle properties and diameter. Thus, a stationary particle layer at the height of $z=0 \mathrm{~m}$ with the same properties like the dispersed particles is adopted. For the $\mathrm{x}$ - and the y-normal surfaces, periodic conditions are applied.

For the properties of the particles, the parameters of Hamburger Sand are adopted. Hamburger sand is a middle to coarse silica sand which offers the advantage of very round grain shapes. The sand density $\rho_{\mathrm{p}}$, Poisson ratio $\nu_{\mathrm{p}}$, coefficient of restitution $e$ and Youngs modulus $E_{\mathrm{p}}$ are determined in the Bachelor thesis of Liebetrau (2013). The coefficient of friction $\mu$ as well as the coefficient of rolling friction $R_{\mu}$ rely on assumptions. Table 3 gives a summary of the DEM properties.

As mentioned in section 4.1 the upstream area of $0.9 \mathrm{~m}$ is applied as a initiation region for the boundary layer flow. Since in this area the velocity distribution is not fully developed, the movement of the particles in this area is not of interest. On this account the particles placed in the first $0.9 \mathrm{~m}$ of the simulation domain are fixed during simulation. To achieve a reduction of computational costs, the fixing is accomplished by eliminating the particles from the integration scheme which is adopted for the update of particle positions and velocities. Nevertheless, the particles are still present and affect the fluid flow.

Table 3: Particle properties of the DEM simulation

\begin{tabular}{lrl}
\hline Parameters & & \\
\hline Density $\rho_{\mathrm{p}}$ & 2639 & $\mathrm{~kg} / \mathrm{m}^{3}$ \\
Coefficient of Restitution $e$ & 0.96 & - \\
Poisson Ratio $\nu_{\mathrm{p}}$ & 0.12 & - \\
Youngs modulus $E_{\mathrm{p}}$ & 2452.90 & $\mathrm{MPa}$ \\
Coefficient of friction $\mu$ & 0.5 & - \\
Coefficient of rolling friction $R_{\mu}$ & 0.4 & - \\
\hline
\end{tabular}

\section{RESULTS}

The fluid flow in the simulations showed the expected development over the channel length. Figure 7 presents the velocity state for a inlet velocity of $0.84 \mathrm{~m} / \mathrm{s}$. In the upstream left half of the simulation domain, the primary uniform velocity distribution from the inlet, is converted under the influence of the particle layer. Thereby, the velocity of the fluid near to the particle layer, becomes slower, while the velocity in the upper parts increases slightly. In the right half, the flow is still influenced but clearly less than in the left half.

During the simulations the fluid velocity is increased stepwise. Thereby, steps of rising velocity and steady velocity alternate, to identify the critical state for the particle transport. Figure 8 shows the top view of a cut-out from the movable particle layer region with $d_{p}=0.8 \mathrm{~mm}$ between $z=0.92 \mathrm{~m}$ and $0.935 \mathrm{~m}$. The particle colour correspond to the particle velocity. Blue particles are currently stationary. Figure 8.1 shows the particle positions right before an initiated movement at $v_{\text {inlet }}=0.76 \mathrm{~m} / \mathrm{s}$. Figure 8.2 represents an increase step to $v_{\text {inlet }}=0.78 \mathrm{~m} / \mathrm{s}$, where the first particle motion occurs. Thereby, just single particles undergo very small motion without explicit transport. The next three figures 8.3 to 8.5 correspond to a steady initial velocity of $v_{\text {inlet }}=0.78 \mathrm{~m} / \mathrm{s}$. As before, just single particles show motion, but additionally transport of these particles over a few mm occurs. All the moved particles are characterized by an exposed initial position without an clear embedding in the layer surface. This state and its indicators are comparable to Kramers second level (weak movement) of visual observation of incipient motion (Kramer 1935).

The last four figures 8.6 to 8.9 represent the particle movement at increasing velocity from $v_{\text {inlet }}=$ $0.80 \mathrm{~m} / \mathrm{s}$ to steady $v_{\text {inlet }}=0.82 \mathrm{~m} / \mathrm{s}$. At first, the number of moved single particles increases. Following by transport of whole particle groups. In the end at figure 8.9 almost the whole topmost particle layer is in 


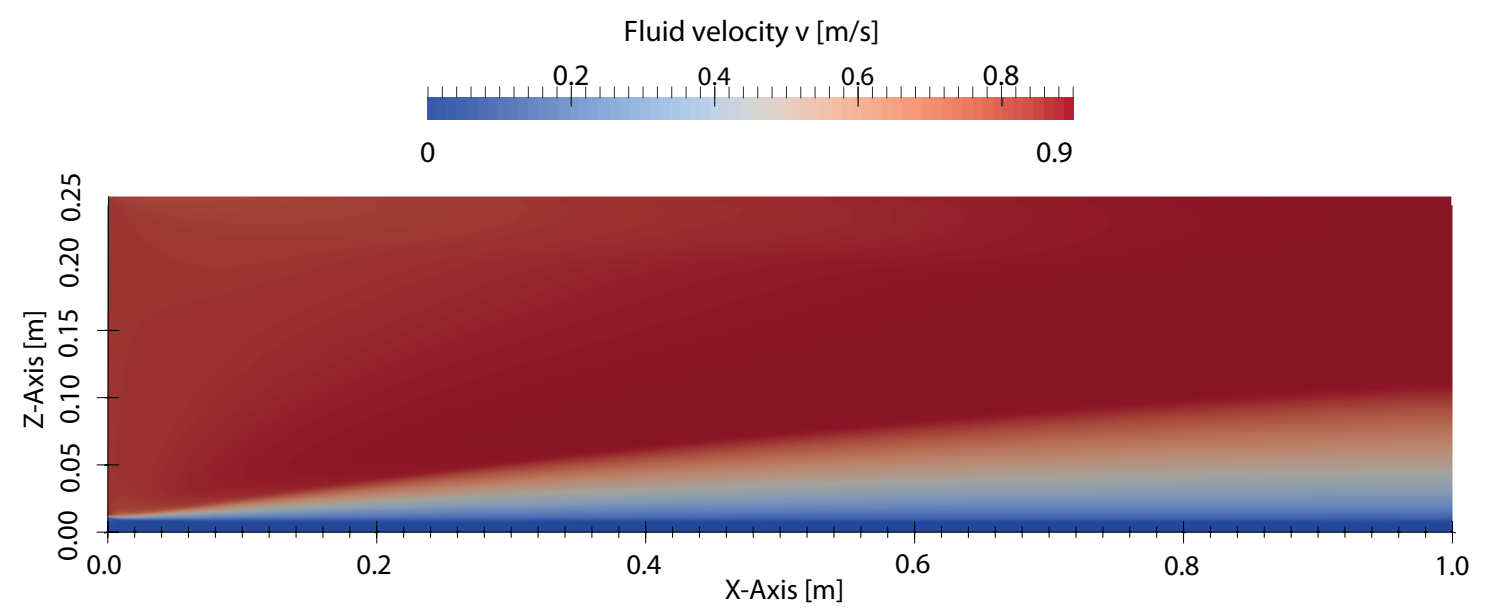

Figure 7: Fluid velocity in the CFD simulation domain for the inlet velocity of $0.8 \mathrm{~m} / \mathrm{s}$

motion and moves in flow direction. At this point, the third level of general transport according to Kramer (1935) occurs. The simulations show the indicative change of bed configuration. Thereby, the upper half of the particle layer rearranges, while the topmost particles are moved in groups in flow direction.
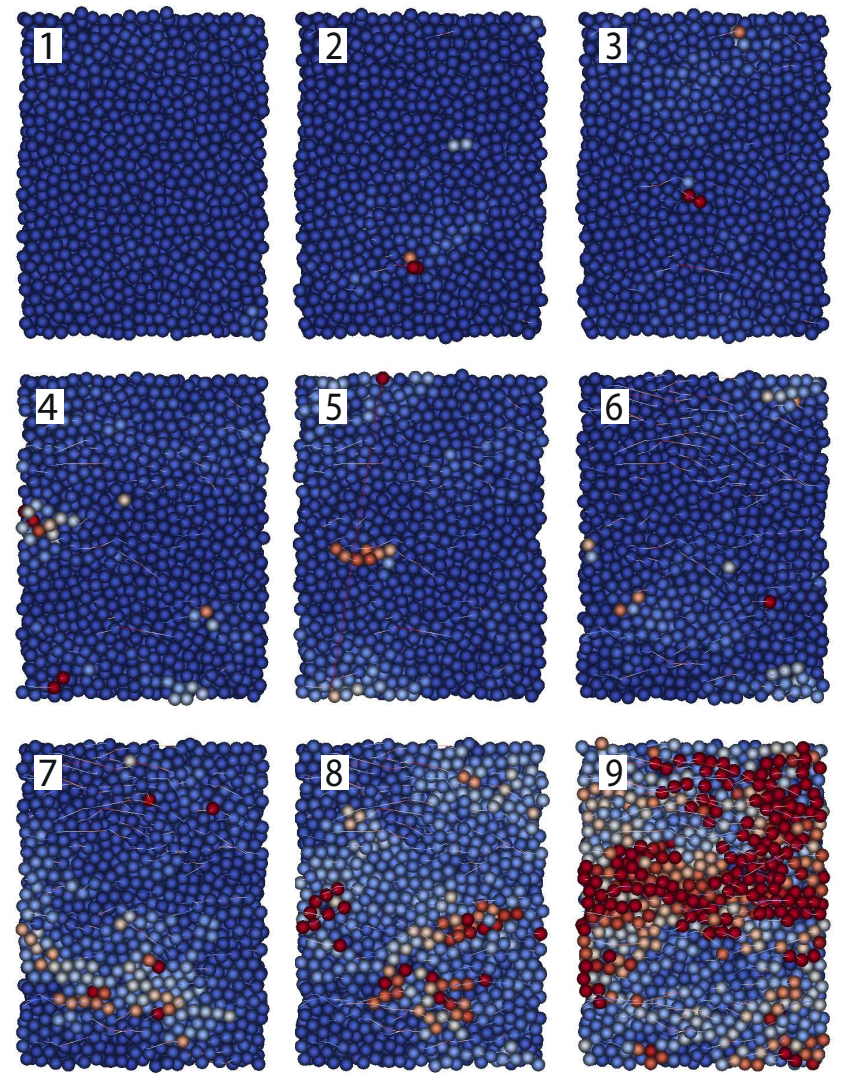

Particle velocity $\mathrm{v}_{\mathrm{p}}[\mathrm{m} / \mathrm{s}]$

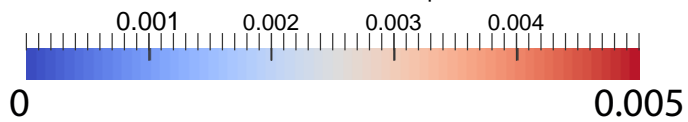

Figure 8: Development of particle movement. Colours according to the particle velocity. Tracing lines describe particle paths

Since the velocity distribution changes over the channel length, the inlet velocity $v_{\text {inlet }}$ is not an appropriate parameter for the characterisation of the flow situation. As a consequence, the inlet velocity $v_{\text {inlet }}$

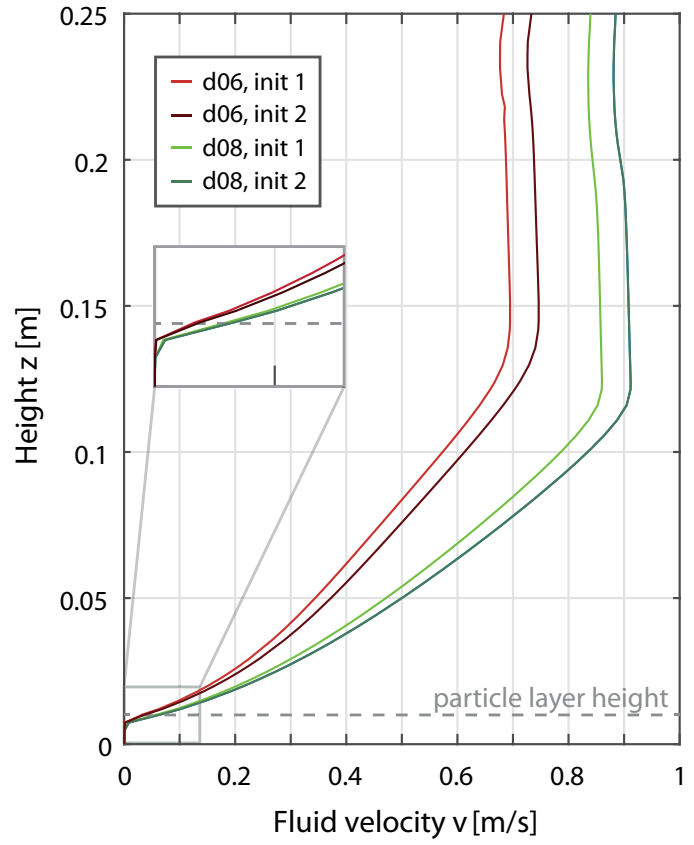

Figure 9: Fluid velocity distribution at initiation of motion for particles with a diameter of $d_{p}=0.6 \mathrm{~mm}$ and $\mathrm{d}_{p}=0.8 \mathrm{~mm}$

does not represent the critical velocity of the initiation of motion. Figure 9 gives the velocity distribution at the transport area located at the position of particle movement. The graph init 1 represents the initiation of single particle transport while init 2 describes the initiation of particle group transport. At the lower diagram area (see detail in figure 9), the influence of the particle layer is visible. Thereby, the velocity distribution reaches into the upper part of the particle layer. While the topmost particles are already in motion due to the flow velocity, the lower particles stay stable at a low filter velocity of approximately $0.01 \mathrm{~m} / \mathrm{s}$. According to the graphs, the single particle transport is initiated at a critical velocity $v_{\text {crit }}$ of approximately $0.70 \mathrm{~m} / \mathrm{s}$ for a monodispersed packing with a diameter $\mathrm{d}_{p}=0.6 \mathrm{~mm}$. The particle group transport starts at a critical velocity of approximately $0.75 \mathrm{~m} / \mathrm{s}$. For a particle diameter of $\mathrm{d}_{p}=0.8 \mathrm{~mm}$ the single particle transport begins at a critical velocity $v_{\text {crit }}$ of $0.85 \mathrm{~m} / \mathrm{s}$, while the group transport is initiated at approximately 


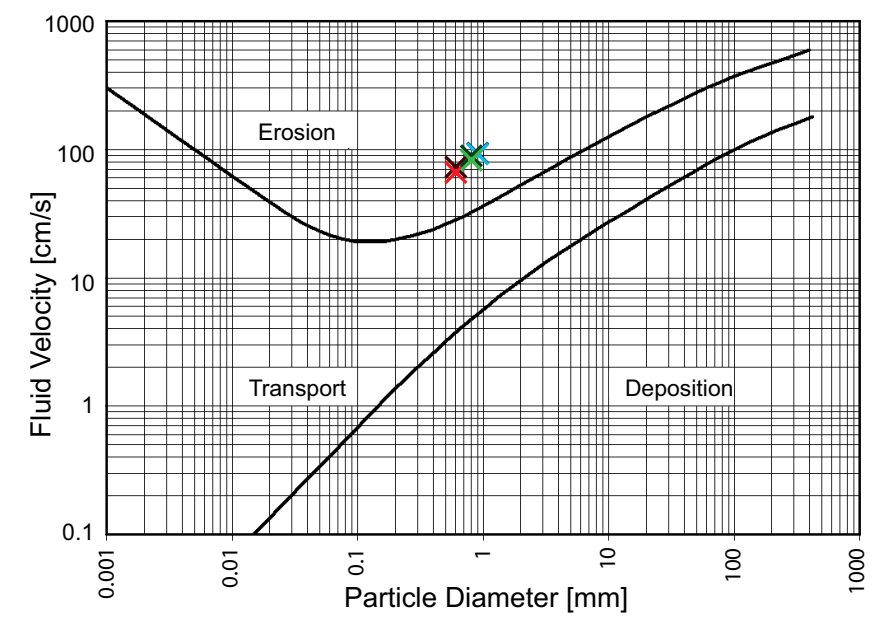

Figure 10: Simulation results for $d_{p}=0.6 \mathrm{~mm}, d_{p}=0.8 \mathrm{~mm}$ and $d_{p}=0.9 \mathrm{~mm}$ implemented in the Hjulström diagram

$0.9 \mathrm{~m} / \mathrm{s}$. Hence, the velocity difference between the single and the group transport amounts approximately $0.5 \mathrm{~m} / \mathrm{s}$ in both cases.

For a approximate verification of the conducted simulations, the resulting critical velocities are implemented into the Hjulström diagram corresponding to the particle diameter (see figure 10). Compared to the approach of Hjulström, the simulation results show clearly higher critical velocities for the initiation of motion for single particle as well as for particle group transport. According to Hjulström, the critical velocity for a particle diameter $d_{p}=0.6 \mathrm{~mm}$ is approximately $0.28 \mathrm{~m} / \mathrm{s}$. For a particle diameter of $d_{p}=0.8 \mathrm{~mm}$ a critical velocity of $0.34 \mathrm{~m} / \mathrm{s}$ is estimated. The critical velocity in the simulations constitute $0.7 \mathrm{~m} / \mathrm{s}$ for a diameter of $0.6 \mathrm{~mm}$ and $0.85 \mathrm{~m} / \mathrm{s}$ for a diameter $0.8 \mathrm{~mm}$. The simulations with a particle diameter of $0.9 \mathrm{~mm}$ show similar results.

\section{CONCLUSION AND DISCUSSION}

The simulations show a realistic behaviour of the initiation of particle motion. Similar to Kramers visual observation of incipient motion, the levels of weak and general particle transport are observable in the simulations. To verify the erosion criteria according to Kramer, the simulations should be implemented with a particle layer consisting of a realistic grain size distribution of Hamburger Sand. Thereby, the effect of multiple grain sizes and the packing density should be investigated.

The simulations show higher critical velocities than the critical values according to Hjulström. This implies, that the fluid flow velocities at the particle surface or the resulting interaction forces are underestimated in the simulations. Although, the resulting flow behaviour seems realistic according to the boundary layer theory of Prandtl (Prandtl 1905), the boundary layer thickness is overestimated in the simulations (see figure 7). Thereby, flow velocities directly at the particle boundary are smaller than in a natural flow regime. As a result, the simulations require higher flow velocities in the outer flow layer to reach the same critical state at the particle surface as a realistic flow distribution.

The calculation of the interaction forces is a central point in the introduced correlation. Hence, the arrangement of the particles with respect to the CFD cells is a main factor for the determination of fluid particle interaction. In the conducted simulations the top edge of the particle layer coincide with the top edge of a cell row. Thus, the value of the particle volume fraction $\alpha_{p}$ is large in this cell row (approximately 0.6 ). The cell row directly above the top edge obtains a particle volume fraction of nearly $\alpha_{p}=0$. To examine this point, the simulations need to be repeated with a varied meshing.

As mentioned in section 3, the turbulence serves as a central point to incipient motion. Turbulence induces an additional velocity fluctuation, whereby the resulting peak values represent the decisive critical conditions for the initiation of motion. Furthermore, the turbulence causes an additional lift force. In the conducted simulations the RANS based k- $\epsilon$ turbulence model is applied. This model considers the mean velocities and determines the energy dissipation resulting from turbulences. The effect of different turbulence models such as the more detailed Large Eddy Simulation (LES) should be examined. The effect of the turbulence induced lift force, should be captured by appropriate model approaches e.g. of Zanke (2003), which is discussed in Goniva et al. (2012).

Further investigations should be carried out to examine the effect of particle friction and rolling friction. Since the values, which are applied in the conducted simulations, are based on assumed data, the effect of these parameters should be tested in physical model tests. Therefore, geotechnical classification test will be applied and compared to equivalent simulations.

Finally, the sediment transport simulations should be validated by small scale model tests. Where in the particle parameters are varied by the usage of different materials, like coarse sand and glass spheres. Furthermore, the effect of the particle layer density should be examined.

\section{REFERENCES}

Bokkers, G. A., M. Van Sint Annaland, \& J. A. M. Kuipers (2004). Comparison of continuum models using the kinetic theory of granular flow with discrete particle models and experiments: Extent of particle mixing induced by bubbles.

Buffington, J. M. (1999). The legend of a. f. shields. Journal of Hydraulic Engineering (125), 376-387.

Crowe, C. T., J. D. Schwarzkopf, M. Sommerfeld, \& Y. Tsuji (op. 2012). Multiphase flow with droplets and particles (2nd ed. ed.). Boca Raton, FL: Taylor \& Francis / CRC Press.

Cundall, P. A. \& R. D. Hart (1992). Numerical modelling 
of discontinua. Engineering Computations 9(2), 101113.

Cundall, P. A. \& O. D. L. Strack (1979). A discrete numerical model for granular assemblies. Géotechnique (29), 47-65.

Dittrich, A. (1998). Wechselwirkung Morphologie/Strömung naturnaher Fließgewässer. Habilitation, Universität Fridericiana zu Karlsruhe, Karlsruhe.

Gidaspow, D. (1994). Multiphase flow and fluidization: Continuum and kinetic theory descriptions. Boston: Academic Press.

Goniva, C., K. Gruber, \& C. Kloss (2012). Sediment erosion a numerical and experimental study. In R. Murillo Muñoz (Ed.), River flow 2012, Leiden, Netherlands, pp. 415-422. CRC Press/Balkema.

Hertz, H. (1881). Ueber die berührung fester elastischer körper. Journal für die reine und angewandte Mathematik (92), 159-171.

Hjulström, F. (1935). Studies of the morphological activity of rivers as illustrated by the River Fyris. Dissertation, Universitet Uppsala, Uppsala.

Hu, G., Z. Hu, B. Jian, L. Liu, \& H. Wan (2011). On the determination of the damping coefficient of non-linear spring-dashpot system to model hertz contact for simulation by discrete element method. Journal of Computers 6(5).

Kloss, C. \& C. Goniva (2011). Liggghts - open source discrete element simulations of granular materials based on lammps. In Supplemental Proceedings, pp. 781-788. Hoboken, NJ, USA: John Wiley \& Sons, Inc.

Koch, D. L. \& R. J. Hill (2001). Inertial effects ins suspension and porous-media flows. Annual Review of Fluid Mechanics 33(1), 619-647.

Kramer, H. (1935). Sand mixtures and sand movement in fluvial models. Transactions of the American Society of Civil Engineers (100(1)), 798-878.

Liebetrau, M.-C. (2013). Untersuchungen zur direkten bestimmung von partikeleigenschaften für die simulationen mit der diskrete-elemente-methode: Bachelorarbeit.

Lu, X., C. M. Boyce, S. A. Scott, J. S. Dennis, \& D. J. Holland (2015). Investigation of two-fluid models of fluidisation using magnetic resonance and discrete element simulations. Procedia Engineering 102, 1436-1445.

McLaughlin, J. B. (1991). Inertial migration of a small sphere in linear shear flows. Journal of Fluid Mechanics 224(-1), 261.

Mei, R. (1992). An approximate expression for the shear lift force on a spherical particle at finite reynolds number. International Journal of Multiphase Flow 18(1), 145-147.

Mindlin, R. D. \& H. Deresiewicz (1953). Elastic spheres in contact under varying oblique forces. Journal of Applied Mechanics (20(1)), 327-344.

Odar, F. \& W. S. Hamilton (1964). Forces on a sphere accelerating in a viscous fluid. Journal of Fluid Mechanics $18(02), 302$.

Plenker, D. (2015). Expansion of cfd-modelling by the cfd-dem method for simulating erosion processes. In J. Grabe (Ed.), Morphodynamics 2015, Volume 33 of Veröffentlichungsreihe des Instituts für Geotechnik und Baubetrieb der TU Hamburg-Harburg, pp. 125-141. Hamburg.
Prandtl, L. (1905). Über flüssigkeitsbewegung bei sehr kleiner reibung. In A. Krazer (Ed.), Verhandlungen des Dritten Internationalen Mathematiker-Kongresses, Heidelberg 1904, pp. 484-491. Leipzig: B. G. Teubner.

Shields, A. F. (op. 1936). Anwendung der Aehnlichkeitsmechanik und der Turbulenzforschung auf Geschiebebewegung. Mitteilungen der Preußische Versuchsanstalt für Wasserbau und Schiffbau. Berlin: Eigenverlage der Preußische Versuchsanstalt für Wasserbau und Schiffbau.

Stein, E., R. d. Borst, \& T. J. R. Hughes (2004). Encyclopedia of computational mechanics. Chichester, West Sussex: John Wiley.

Tsuji, Y., T. Tanaka, \& T. Ishida (1992). Lagrangian numerical simulation of plug flow of cohesionless particles in a horizontal pipe. Powder Technology 71(3), 239-250.

Zanke, U. (2001). Zur Einfluss der Turbulenz auf den Beginn der Sedimentbewegung, Volume Heft 120 of Mitteilungen. Darmstadt: Technische Universität Darmstadt.

Zanke, U. (2003). On the influence of turbulence on the initiation of sediment motion. International Journal of Sediment Research (18), 17-31.

Zhou, Z. Y., S. B. Kuang, K. W. Chu, \& A. B. Yu (2010). Discrete particle simulation of particle-fluid flow: Model formulations and their applicability. Journal of Fluid Mechanics 661, 482-510.

Zhu, H. P., Z. Y. Zhou, R. Y. Yang, \& A. B. Yu (2007). Discrete particle simulation of particulate systems: Theoretical developments. Chemical Engineering Science 62(13), 3378-3396. 